\title{
The challenges of GALEX
}

Karl Forster, Tim Conrow, David Schiminovich, Kerry D. Erickson, Justin F. McNeill, et al.

Karl Forster, Tim Conrow, David Schiminovich, Kerry D. Erickson, Justin F. McNeill, Chris Martin, Marco Morais, "The challenges of GALEX," Proc. SPIE 6270, Observatory Operations: Strategies, Processes, and Systems, 627004 (29 June 2006); doi: 10.1117/12.672397

Event: SPIE Astronomical Telescopes + Instrumentation, 2006, Orlando, Florida , United States 


\title{
The challenges of GALEX
}

\author{
Karl Forster, ${ }^{a}$ \\ Tim Conrow, ${ }^{a}$ David Schiminovich, ${ }^{b}$ Kerry D. Erickson, ${ }^{c}$ \\ Justin F. McNeill Jr, ${ }^{d}$ Chris Martin, ${ }^{a}$ and Marco Morais ${ }^{d}$ \\ ${ }^{a}$ California Institute of Technology, 1200 E. California Blvd., Pasadena, CA 91125 \\ ${ }^{b}$ Columbia University, 550 W. 120th St., New York, NY 10027 \\ ${ }^{c}$ Jet Propulsion Laboratory, 4800 Oak Grove Drive, Pasadena, California 91109 \\ ${ }^{d}$ The Aerospace Corp., 2350 E. El Segundo Blvd., El Segundo, CA 90245
}

\begin{abstract}
The Galaxy Evolution Explorer, a NASA small explorer mission, is performing the first all-sky, deep imaging and spectroscopic surveys in the space ultraviolet. The prime goal of GALEX is to study star formation in galaxies and its evolution with time. Now in its fourth year of operations the emphasis of the mission is changing from completing the primary science goals set at launch to servicing the astronomical community with a guest investigator program that uses $50 \%$ or more of the available observing time. We outline here mission operations, describe some of the challenges the GALEX team has surmounted, and some of the changes needed to accomplish the goals of the extended mission.
\end{abstract}

Keywords: galaxies:evolution - space vehicles:instruments - surveys - telescopes - operations - ultraviolet:general

\section{INTRODUCTION}

The Galaxy Evolution Explorer (GALEX) is a NASA small explorer class mission (SMEX) launched on April 28 2003. In the first three years of operations GALEX is achieving the objective of tracing the global star formation history of the universe by performing the first all-sky imaging and spectroscopic surveys in the space ultraviolet $(1350-2750 \AA)$. Although designed to explore the specific questions associated with the evolution of star formation history in galaxies over the redshift range $0<\mathrm{z}<2(\sim 7$ billion years), GALEX is also well suited to investigate the ultraviolet (UV) properties of objects in the solar system and the milky way galaxy. More detailed presentation of the successes of the GALEX science mission are presented in Martin (2006). ${ }^{1}$

The primary mission was designed to be achieved in 28 months of science operations but the popularity and success of observations has resulted in recommendations from NASA science review panels that the GALEX mission be extended to 2010. The addition of a guest investigator program to the primary science surveys has provided a challenge to mission operations that will be discussed here. The longevity of the GALEX mission also poses (very welcome) challenges to instrument and spacecraft operations that is being met with major upgrades to the mission planning system, operations procedures, and the pipeline processing of the large volume of GALEX data. As with any operating space mission the response to spacecraft and instrument anomalies is another challenge to the small, dedicated, and knowledgeable GALEX team. The success of the adjustments to mission operations in response to these anomalies are discussed here.

\section{MISSION OVERVIEW}

\subsection{Science Instrument}

The GALEX instrument allows imaging and spectroscopic observations to be made in two ultraviolet bands, Far

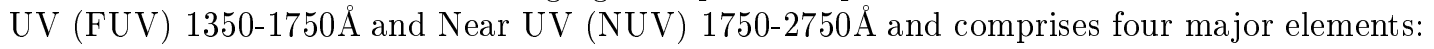

Further information from Karl Forster: E-mail krl@srl.caltech.edu, Telephone: 16263952101

Observatory Operations: Strategies, Processes, and Systems, edited by David R. Silva, Rodger E. Doxsey, Proc. of SPIE Vol. 6270, 627004, (2006) · 0277-786X/06/\$15 - doi: 10.1117/12.672397 


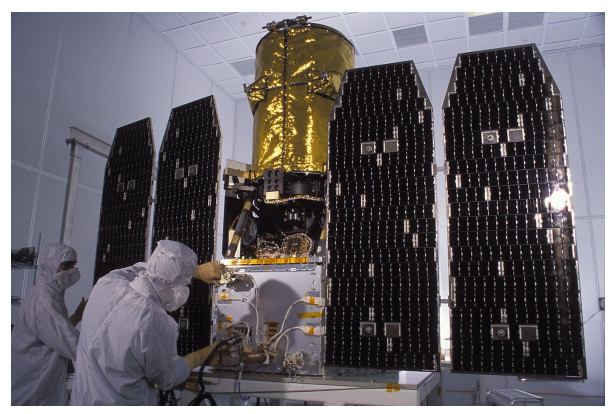

Figure 1. GALEX instrument and spacecraft with solar panels deployed.

1. A modified Richey-Chrétien telescope of $50 \mathrm{~cm}$ diameter with a 3 meter focal length and $1.2^{\circ}$ circular field of view.

2. An optical wheel assembly with two apertures, a $\mathrm{CaF}_{2}$ imaging window, and a $\mathrm{CaF}_{2}$ transmission grism. The grism can also be rotated to provide multiple dispersion angles to remove spectrum overlap issues. An opaque position is also used to block light from entering the back focal assembly.

3. A dichroic beam splitter that reflects the FUV and transmits the NUV band.

4. Two, sealed tube, microchannel plate detectors with $65 \mathrm{~mm}$ active area and grid delay-line anode that allow photon event positions and times ( $\sim 5$ msec accuracy) to be recorded.

The instrument provides simultaneous co-aligned FUV and NUV images with spatial resolution 4.5 and 5.1 arcsec respectively. The grism mode provides low resolution spectra (R FUV/NUV 200/100) of all objects in the field of view. Details of the performance of the instrument and detectors can be found in Martin et al. $(2005)^{2}$ and Morrissey et al. (2005). ${ }^{3}$

\subsection{Spacecraft}

GALEX was launched by a Pegasus-XL vehicle on April 282003 into a $690 \mathrm{~km}$ altitude, $29^{\circ}$ inclination, circular orbit with a 98.6 minute period. The projected orbit lifetime is more then 25 years and the solar array power margin is expected to remain above $30 \%$ until 2010 with useful battery life through 2015 . There are no consumables on board. The spacecraft is three axis stabalized with selective redundancy in the gyros and reaction wheels. No flight hardware failures have occured.

The mission design is straightforward. Science data is only collected on the night side of the orbit with the instrument boresite pointing at the science target and performing a spiral dither motion of 1.5 arcminute diameter. This is done to reduce detector fatigue by bright stars. On the day side of each orbit the spacecraft is aligned with solar panels oriented toward the Sun and the instrument boresite pointed away from the Earth (see Fig. 2). The length of the detector exposures on the night side ranges from 15 to 28 minutes. The voltage level of the detectors are ramped to low values during the day side of each orbit to protect detectors from damage. The detectors are also held at low voltage during spacecraft passages through the South Atlantic Anomaly (SAA). When this occurs on the night side of an orbit the exposure times are reduced (see Fig. 3), occasionally causing no exposure to be taken.

Photon data and housekeeping instrument and spacecraft telemetry are stored on the spacecraft solid state recorder (SSR) which has a capacity of 3 GB. Portions of the SSR are played back during ground station contacts via an X-band (25 Mbps) transmitter. An S-band transmitter is also available for spacecraft telemetry (2 Mbps) and spacecraft commanding is through a 2 kbps S-band link. 


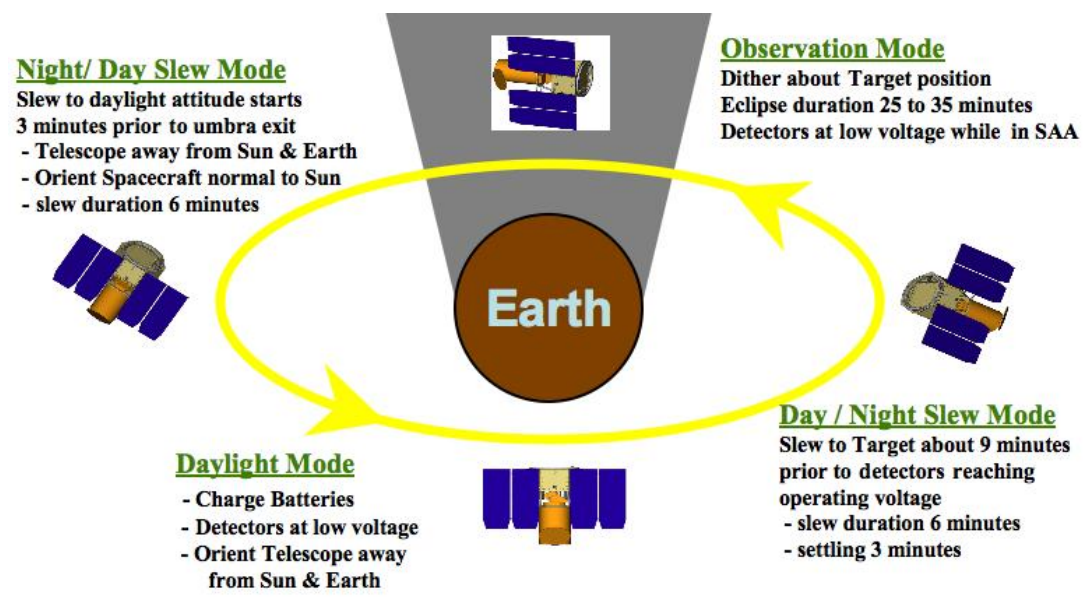

Figure 2. GALEX orbit attitude. Exposures are only taken on the night side of the orbit.

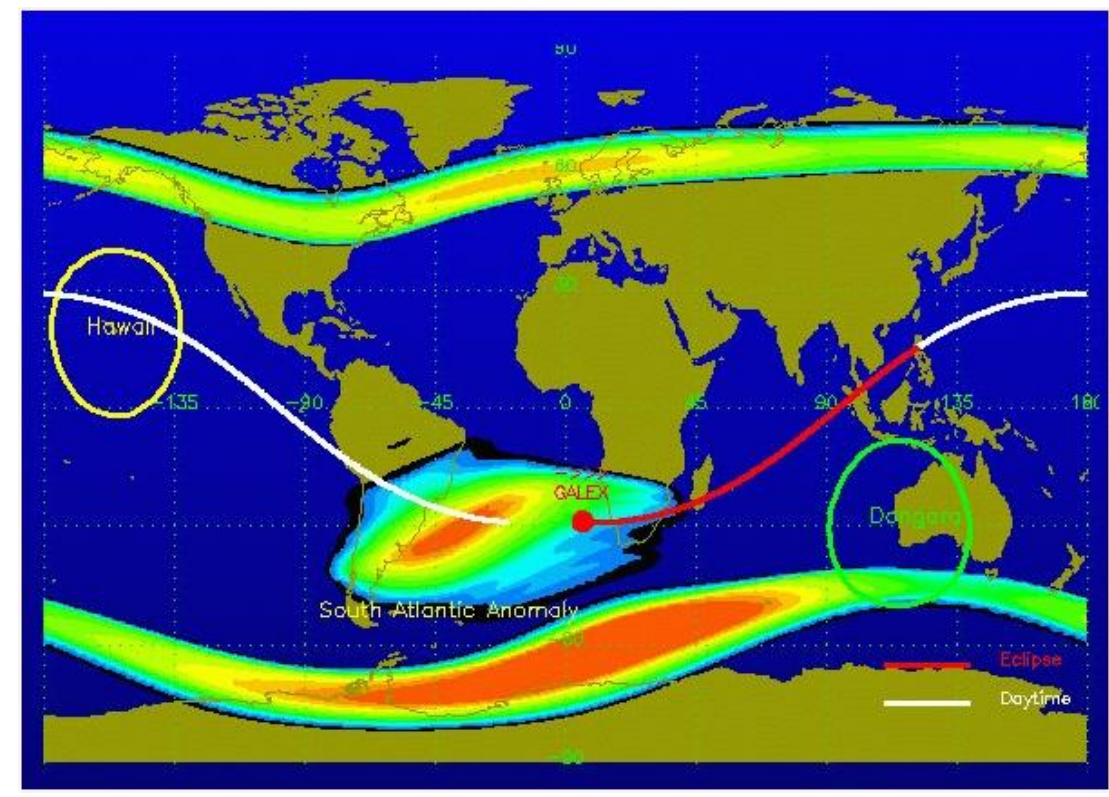

Figure 3. Ground trace of a single GALEX orbit. The particle flux associated with the SAA is modeled (blue = low to red=high). The red line is the night side of the orbit, the day side is white. The high voltage supply on the detectors are not ramped until after exiting the SAA. The viewing cones of the two ground stations are also displayed. A contact with the Hawaii station is possible on this orbit.

\subsection{Operations}

Mission operations are managed by orbital sciences corporation (http://www.orbital.com) based in Dulles VA. (MOC) and science operations are managed by the GALEX team based at the California Institute of Technology in Pasadena, CA. (SOC). Universal Space Network (http://www.uspacenetwork.com/) provides communications with the spacecraft using ground stations in Hawaii and Dongara, Australia. Fig. 4 shows a schematic of the operations between the MOC, SOC, and the spacecraft. Real time telemetry is received during spacecraft contacts at the MOC and relayed to the SOC. Commands are sent from the MOC to the spacecraft, each contact lasting between 5 and 12 minutes. GALEX also has a TDRSS capability for use in spacecraft emergencies.

GALEX is designed to operate autonomously using a sequence of commands (ATS) lasting 9 days uploaded 


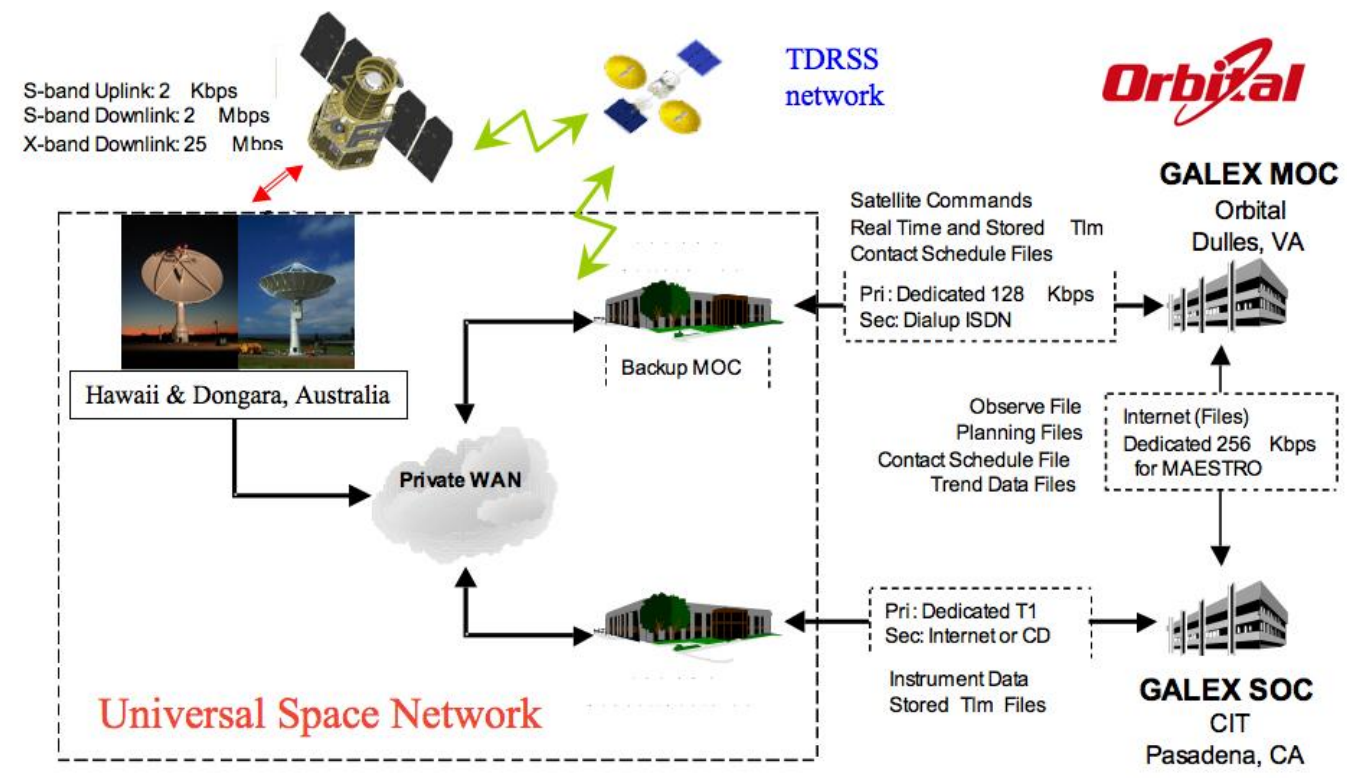

Figure 4. GALEX mission operations schematic.

once per week. The science targets for each plan are chosen by the SOC and a preliminary observation plan is then passed to the MOC where the day side attitude and power requirements are examined. The on-board ATS buffer has two partitions and each can hold 2600 commands. New ATS are loaded into the inactive buffer and then switched to during a period of command inactivity. Ground station contacts are selected by the SOC to enable data stored in the SSR to be relayed to the ground and space on the SSR freed for recording new data. The schedule of contacts is chosen so that the SSR does not reach capacity. This usually requires 3 contacts per $24 \mathrm{hr}$ day but is dependent on the UV brightness of the science targets which can result in up to 130,000 photon events being recorded every second.

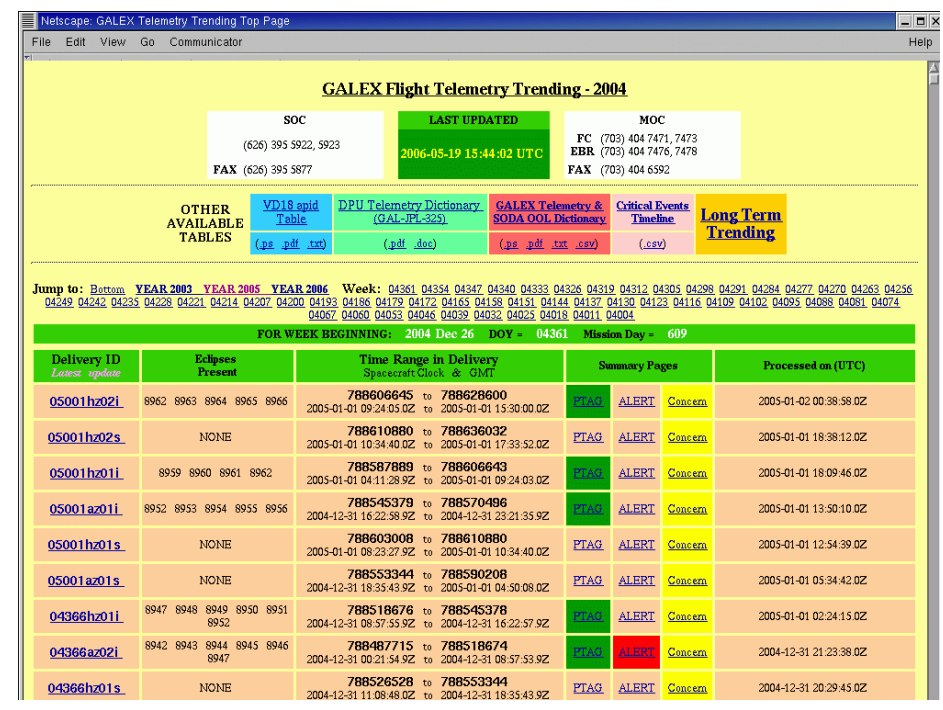

Figure 5. GALEX telemetry trending webpage (top level). 
The SSR has separate partitions for spacecraft housekeeping telemetry (VC18) and detector photon and instrument housekeeping telemetry (VC32). The sections of each partition played back on every scheduled contact are delivered to the SOC, VC18 arriving within an hour and VC32 within 8 hours of the contact time. This means that the maximum time between an observation and the availability of fully processed images and flux calibrated source catalogs will be $\sim 24$ hours. Housekeeping telemetry will have at most a latency of 8 hours and is automatically trended as part of the pipeline process. Webpage access to telemetry trending plots and analysis are available for the team to study (see Figs. 5 and 6) and alert email and pager messages are automatically sent out when any telemetry point is out of limits. This is a backup to the alert system at the MOC which sends messages to the team if any telemetry point is observed to be out of limits during a contact.

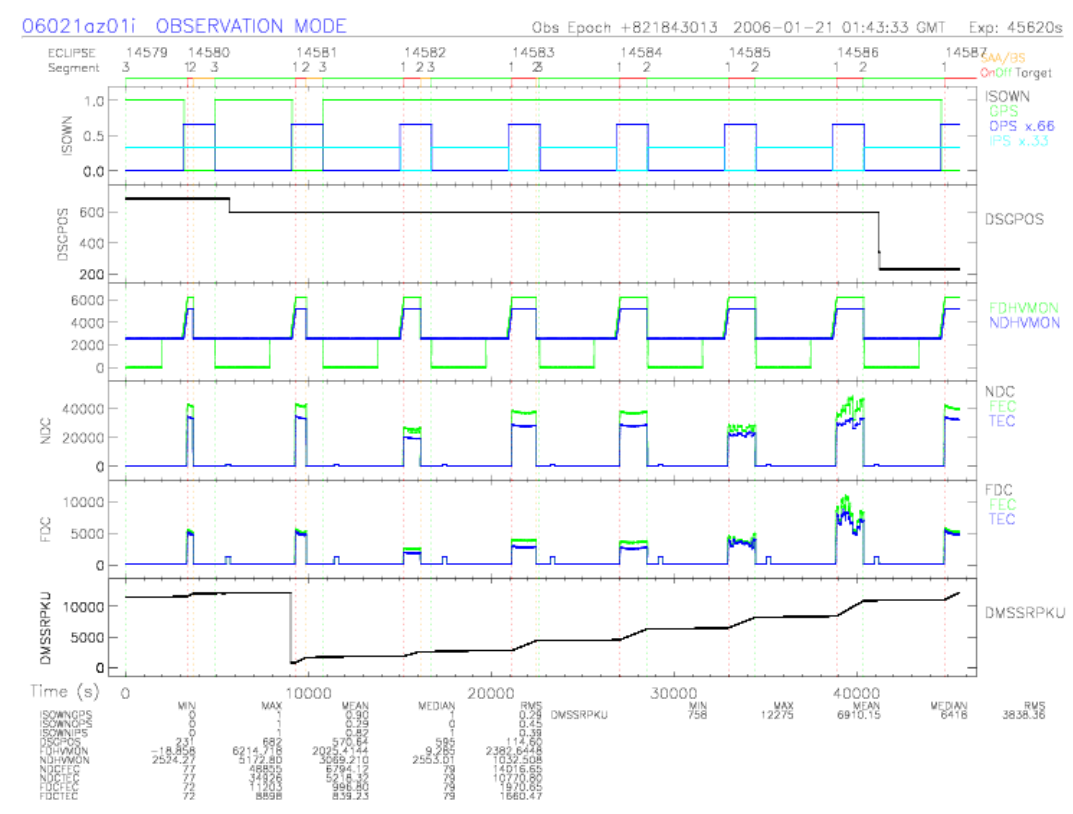

Figure 6. One of the GALEX telemetry trending plots. This plot shows (top to bottom plot stripes) the motion of the optical wheel, the rotation of the grism, the voltage level of the detectors, NUV count rates, FUV count rates, and SSR loading. Note that eclipses 14580 to 14583 are shortened by passage through the SAA during eclipse and that a contact occurred just before eclipse 14581 allowing space to be freed on the SSR.

\section{CHALLENGES}

\subsection{Data volume}

The average raw data volume downloaded from the SSR is $3.2 \mathrm{~GB} /$ day. Upon delivery of the raw data to the SOC a pipeline processing system trends housekeeping telemetry and analyzes detector photon events to produce 14 GB of images and catalogs including 2000 FITS files. Data processing includes: rectifying and computing pointing from time tagged photon lists; image reconstruction; flagging or masking instrument artifacts; flat fielding; astronomical background subtraction; noise estimation; source extraction; source shape, photometric, and astrometric parameter estimation; flux calibration; co-adding repeated observations; and merging FUV and NUV bands. For grism observations, multiple exposures are stacked and individual spectra are extracted, band combined and calibrated.

All data is examined by the SOC as part of an extensive quality assurance (QA) program before being released to the public through the Multi-mission Archive at Space Telescope Science Institute (MAST http://galex.stsci.edu/GR2).

The challenge for GALEX has not only been the complexity of data processing but the volume of data (see Fig.7). Improvements in both hardware (CPU, disk volume, and network) and software (pipeline processing, 


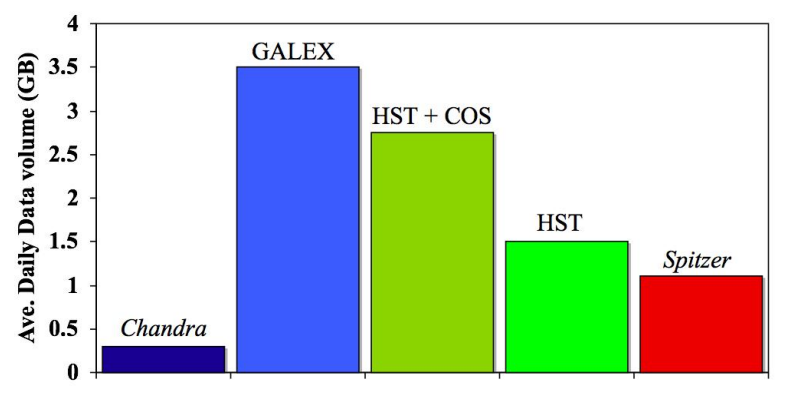

Figure 7. GALEX daily data volume compared to NASA Great Observatories. Data volume for observatories is from Chandra Estes et al. (2000) ${ }^{4}$ HST Pollizzi $(1995) ;{ }^{5}$ Spitzer Werner et al. (2004). ${ }^{6}$

QA system) during the mission have removed bottlenecks in the processing of new data and the re-processing of large amounts of data required for the annual data releases. The ability to reprocess data has grown by a factor of ten since launch and the SOC now has the ability to reprocess one year of raw data $(\sim 1$ TB) in two weeks. This is important as the size of the annual data releases will grow each year:

Table 1. GALEX Data Release to MAST

\begin{tabular}{c|c|c|c|c} 
& $\begin{array}{c}\text { Total } \\
\text { exposure } \\
\text { Release }\end{array}$ & $\begin{array}{c}\text { Total } \\
\text { area } \\
\text { (Sq.Deg.) }\end{array}$ & $\begin{array}{c}\text { Catalog } \\
\text { objects } \\
\text { (million) }\end{array}$ \\
\hline Early data release & 2004 & 48 & 10 & $\sim 0.05$ \\
GR1 & 2005 & 1300 & 3000 & $>10$ \\
GR2 & 2006 & 4000 & 7000 & $>30$ \\
GR3 (projected) & 2007 & 15000 & 30000 & $>200$
\end{tabular}

\subsection{Evolving science surveys}

The science goals of the GALEX mission are achieved with a series of nested surveys, i.e. observations of many survey types are made each day, although only 1 survey type can be assigned to each eclipse. The mission planning system (MPS) was designed to achieve the goals of the GALEX mission in 28 months of science operations using a fixed set of targets chosen before launch. This allowed for some flexibility in mission planning, a target that did not reach the exposure goal in 2004 could be observed again in 2005. However that addition of a Guest investigator program (GIP) in 2004 required a change in the way targets were assigned to eclipses, which must now be carefully monitored to make sure that the exposure goals for GIP targets are achieved within each cycle. Figure 8 gives an indication of the changing mix of surveys throughout the mission and the proposed additions for the extended mission. Parameters that have changed since launch are highlighted in red.

The major changes to the GALEX surveys include:

Detector count rate limit adjustments - The high sensitivity of the GALEX detectors means that observations of stars brighter the $m_{B} \sim 9.0$ may damage the detectors. Operations before October 2005 were made with a conservative count rate limit for the NUV detector of $5000 \mathrm{cts} / \mathrm{s}$ for single sources and this reduced the area available to the All-sky Imaging Survey (AIS) to $\sim 18,000$ square degrees. Investigations of the level of detector fatigue for stars brighter then this limit were performed in the summer of 2005 and the count rate limit for the NUV detector was relaxed to $30,000 \mathrm{cts} / \mathrm{s}$. This increases the available sky for the AIS to $\sim 30,000$ square degrees. Further count rate limit adjustments will be necessary to achieve the original goal of mapping the whole ultraviolet sky. 


\begin{tabular}{|c|c|c|c|c|c|c|c|c|c|}
\hline & \multirow[b]{2}{*}{ Survey } & \multicolumn{2}{|c|}{2003 Launch } & \multicolumn{2}{|l|}{ 2004-05 } & \multicolumn{2}{|l|}{2006} & \multicolumn{2}{|l|}{$\begin{array}{c}2007-10 \\
\text { Extended Mission }\end{array}$} \\
\hline & & $\begin{array}{c}\text { Area } \\
\text { (Sq. Deg.) }\end{array}$ & $\begin{array}{l}\text { Exp } \\
\text { (ks) }\end{array}$ & $\begin{array}{c}\text { Area } \\
\text { (Sq. Deg.) }\end{array}$ & $\begin{array}{l}\text { Exp } \\
\text { (ks) }\end{array}$ & $\begin{array}{c}\text { Area } \\
\text { (Sq. Deg.) }\end{array}$ & $\begin{array}{l}\text { Exp } \\
\text { (ks) }\end{array}$ & $\begin{array}{c}\text { Area } \\
\text { (Sq. Deg.) }\end{array}$ & $\begin{array}{l}\text { Exp } \\
(\mathrm{ks})\end{array}$ \\
\hline \multirow{4}{*}{ 声 } & All-sky & $\begin{array}{c}41,000 \\
\text { Scan-mode }\end{array}$ & 0.1 & $\begin{array}{c}18,000 \\
\text { Chain dither }\end{array}$ & 0.1 & $\begin{array}{c}30,000 \\
\text { New count rate limit }\end{array}$ & 0.1 & $\begin{array}{c}38,000 \\
\text { New Count rate limit? }\end{array}$ & 0.1 \\
\hline & Deep & 80 & $\begin{array}{c}30 \text { to } \\
150\end{array}$ & 80 & 30 & 80 & 30 & +100 & 30 \\
\hline & Medium & 1000 & 1.5 & 1000 & 1.5 & 1000 & 1.5 & +8500 & 1.5 \\
\hline & Nearby Galaxy & 200 & 2.0 & 300 & 2.0 & 300 & 2.0 & & \\
\hline \multirow{3}{*}{ 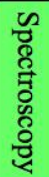 } & Wide-field & 80 & 30 & 80 & 30 & & & & \\
\hline & Medium & 8 & 300 & 8 & 300 & 8 & 300 & +20 & 150 \\
\hline & Deep & 2 & 1500 & & & & & & \\
\hline \multirow{2}{*}{\multicolumn{2}{|c|}{ Guest Investigator }} & & & cycle 1 & $0.1-$ & cycle 2 & 0.1 - & cycles 3-6 & - \\
\hline & & & & $30 \%$ allocation & 80 & $30 \%$ allocation & 65 & $50 \%$ allocation & 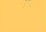 \\
\hline \multicolumn{2}{|c|}{ Planning Cycle } & \multicolumn{2}{|c|}{2 months } & \multicolumn{2}{|l|}{ weekly } & \multicolumn{2}{|l|}{ weekly } & \multicolumn{2}{|l|}{ Bi-weekly } \\
\hline
\end{tabular}

Figure 8. Evolution of the GALEX science surveys.

Spectroscopic surveys - Experience with analysis using the spectroscopic survey data resulted in the concentration of the spectroscopic surveys to the Medium Spectroscopic Survey.

Nearby Galaxy Survey - The Nearby Galaxy Survey (NGS) was expanded after the discovery of extended star formation in GALEX NGS images.

Guest Investigator Program - GALEX is the only SMEX to offer a Guest Investigator Program and community interest is strong, with an over-subscription rate of 3.5 for cycles $1 \& 2$. The SOC supports the GIP by performing technical reviews of the proposals, documentation for website (http://galexgi.gsfc.nasa.gov/) as well as the design and scheduling of GIP observations, processing of data, and regular delivery of GIP data to MAST. The variety of astronomical objects observed as part of the GIP has broadened the immediate science return of GALEX and to achieve this the MPS system has expanded. New modes of operation and planning include:

Target of Opportunity observations, including comets, supernovae and variable stars.

Coordinated observations with ground and orbiting observatories (e.g. Deep impact encounter with Comet Tempel 1).

Observations timed at specific intervals to examine stellar variability.

Time domain Survey - Future observations designed to achieve the PI science goals of the extended mission will also be part of a broad survey examining UV variability of the sky. GALEX can sample variability on timescales of $1 \mathrm{msec}$ to years and will provide variable object alerts for other observatories to follow up. This requires a major upgrade to the GALEX MPS to allow specific timing of observations for all surveys.

Further improvements to the GALEX MPS will include relaxation of the boresite avoidance angle limits to the Earth and Moon, adjustments to the SAA model to maximize the exposure time available, and possibly the inclusion of day side FUV observations. 


\subsection{Anomaly Response}

The GALEX team has responded to a number of instrument and spacecraft anomalies in the first three years of operations. Adjustments to the MPS and to operations procedures have been made and operation efficiency has reached $95 \%$. The major anomalies have been:

FUV detector window charging - Solar activity has caused build-up of charge on the FUV detector window and resulted in detector shutdowns that required more then a week for the detector to recover. The MPS and on-board DPU software has been improved to allow the high voltage of the detector to be turned off on the day side of each orbit. This appears to have eliminated the problem. Details can be found in Morrissey (2006). ${ }^{7}$

FUV detector current anomaly - Imperfections in one of the three microchannel plates in the FUV detector have caused shutdowns of the detector. This first occurred in March 2005 and the recovery procedure lasted 6 months. The recovery involves rapid cycling of the high voltage (HV) supply to the FUV detector with increasing voltage limits. The MPS and on-board software have now been improved to allow for many $\mathrm{HV}$ cycles each orbit and the detector was brought back to nominal observations in 2 weeks after the most recent anomaly in March 2006. Details can be found in Morrissey (2006). ${ }^{7}$

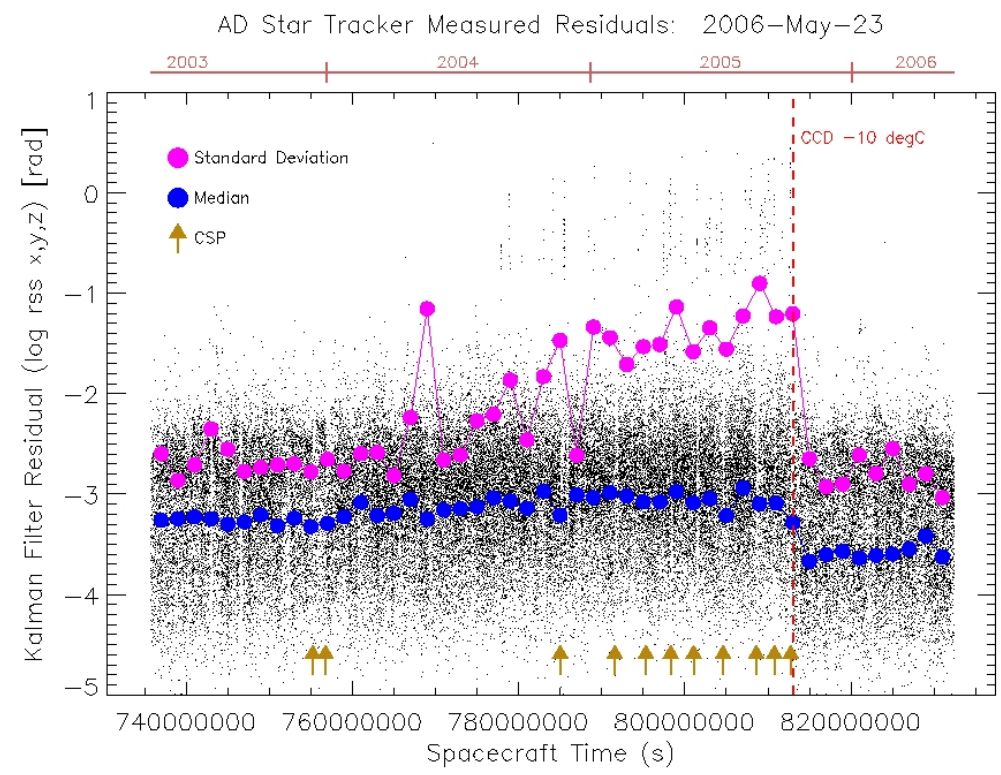

Figure 9. GALEX Star Tracker Kalman filter residuals. Median residuals increased from 1.9 arcsec to 3.2 arcsec before the CCD temperature adjustment and are now $\sim 0.8$ arcsec. The time of CSP entry events are marked and are linked to residuals $>3^{\circ}$.

Star Tracker CCD - GALEX uses a single Ball Aerospace CT-633 star tracker (ST) to provide attitude determination and control. In December 2003 the spacecraft fault detection \& correction responded to unusually high ST residuals by entering Coarse Sun Pointing (CSP) mode. This required 2 weeks to recover to nominal operations. Between February 2005 and October 2005 the star tracker residuals steadily degraded and GALEX entered CSP a further 10 times. It was determined that hot spots on the ST CCD had appeared causing the ST software to confuse the hot spots with stars. On October 112005 the operating temperature of the ST CCD was lowered from $0^{\circ} \mathrm{C}$ to $-10^{\circ} \mathrm{C}$. This resulted in an immediate, and significant improvement in the science data pointing. The ST has performed very well since then with no CSP events (see Fig. 9). Improvements made to the data processing pipeline have also allowed $98 \%$ of exposure time with poor pointing stability to be recovered. 


\section{SUMMARY}

All GALEX flight and ground systems are healthy and indicate that the mission can extend past 2010. The excellent FUV and NUV data meet or exceed pre-launch expectations and all instrument and spacecraft anomalies have been resolved. Although GALEX is a relatively simple, single instrument, it faces an enormous observation planning challenge, with two wide field detectors, imaging and spectroscopic modes, multiple surveys and a Guest Investigator Program with widely varying planning constraints. Each member of the GALEX team has multiple tasks, not only in mission planning and operations, but also as members of a broader GALEX science team that is producing an increasing number of papers including 2 dedicated issues of Astrophysical Journal Letters and Supplement. The challenge of operating GALEX is being met with enthusiasm and dedication by this small team.

\section{ACKNOWLEDGMENTS}

The authors gratefully acknowledge the support of NASA for construction, operation, and science analysis of the GALEX mission, developed in cooperation with the Centre National d'Etudes Spatiales of France and the Korean ministry of Science and Technology. We acknowledge the dedicated team of engineers, scientists, and administrative staff from Caltech/JPL, Orbital Sciences Corporation, UC Berkeley, Laboratory Astrophysique Marseille, and other institutions that made this mission possible.

\section{REFERENCES}

1. C. Martin, "The galaxy evolution explorer," in Astronomical Telescopes and Instrumentation: Space Telescopes and Instrumentation II: Ultraviolet to Gamma Ray, Proc. SPIE, 2006.

2. D. C. M. et al., "The galaxy evolution explorer: A space ultraviolet survey mission," ApJL 619, pp. L1-L6, 2005.

3. P. M. et al., "The on-orbit performance of the galaxy evolution explorer," ApJL 619, pp. L7-L10, 2005.

4. A. E. et al., "The chandra xray center data archive interfaces," in ADASS IX, D. C. N. Manset, C. Veillet, ed., ASP Conf. Proc. 216, p. 457, 2000.

5. J. P. III, "The hubble data archive: Opening the treasures of the hst to the community," in ADASS IV, J. H. R.A. Shaw, H.E. Payne, ed., ASP Conf. Ser. 77, p. 162, 1995.

6. M. W. et al., "The spitzer space telescope mission," ApJS 154, p. 1, 2004.

7. P. Morrissey, "A galex instrument overview and lessons learned," in Astronomical Telescopes and Instrumentation: Space Telescopes and Instrumentation II: Ultraviolet to Gamma Ray, Proc. SPIE, 2006. 